\title{
Book Review: E. Martín-Monje, I. Elorza, I. and B. García Riaza (eds) (2016). Technology-Enhanced Lan- guage Learning for Specialized Domains. Practical applications and mobility. New York: Routledge, pp. 286, ISBN: 978-1-315-65172-9.
}

\section{Salvador Montaner Villalba ${ }^{1}$}

Valencian International University

Computers have had a significant presence in language teaching since the 1960s, while the obvious emerging development of "educational technology" can be established in the early 1980s. By then, this term began to obtain significant popularity, since instructional media started to get a wider impact on educational practices. Since then, terminology has shifted significantly, from the initial Computer-Assisted Language Learning (CALL) to Technology-Enhanced Language Learning (TELL), subtly considering the fact that present computers are transforming less obvious "on the surface" while, at same time, being completely necessary. Computers lead other kinds of technology, such as audio, video and the World Wide Web, so that the current focus is on the communication which is facilitated by the computer rather than the machine itself.

The book Technology-Enhanced Language Learning of Specialized Domains. Practical application and mobility is the latest reference in educational technology and languages for specific purposes (LSP), being preceded by the publication of Arnó Macia, Soler Cervera and Rueda Ramos (2006) and offers readers current research

\footnotetext{
Corresponding author - Faculty of Education, English Language Teaching \& Teacher Training. Valencian International University, Valencia (Spain).

Email: salvador.montaner@campusviu.es
} 
and learning tools to implement in the classroom. The present volume is rich in primary research and empirical studies of twenty high-quality articles, written by 32 authors who are international experts in the field. These authors research into the ongoing methodological and technological (r)evolution within computer-assisted and technology-enhanced language learning. This book, edited by Elena MartínMonje, Izaskun Elorza \& Blanca García Riaza, intends to explore the latest developments in technology-enhanced learning and the processing of languages for specific purposes. It combines both theoretical and applied research from an interdisciplinary angle, including general issues dealing with learning languages with computers, assessment, Mobile Assisted Language Learning, Language Massive Online Open Courses, corpus linguistics and technology-enhanced translation.

This book features an introduction, in which the editors explain how each of the 20 chapters fit into the six thematic sections. Each thematic section contains up to four chapters. As far as the content of each section is concerned, this is distributed this way: Firstly, Part 1 takes the reader, in a very attractive way, right into the complexity of some significant considerations about computer-assisted language learning. Secondly, Part 2 focuses on the role of technology enhanced assessment in language learning, whereas Part 3 offers a wide overview into how mobile-assisted language learning can be applied within processing language for specialized domains. Next, Part 4 explores the didactic applications of language MOOCs, continuing with Part 5 which pays special attention to the effects of corpus-based approaches to specialized linguistic domains and, finally, this appealing volume concludes with Part 6 which examines the role of computer-assisted translation tools in language learning.

Part 1 contains four chapters, two of which deal with the wide topics of digital literacy (Languages and literacies for digital lives written by Mark Pegrum) and developing intercultural competence through telecollaboration (Promoting intercultural competence in culture and language studies: Outcomes of an international collaborative project, by Margarita Vinagre). Mark Pegrum, in his chapter on digital literacy, discusses the globalized world in which language teachers face not only various types of responsibilities for teaching languages and cultures, but also multiple literacies or multiliteracies. In the second chapter by Margarita Vinagre on developing intercultural competence, the author researches in telecollaboration giving a significant relevance to the potential of stimulating learners' intercultural competence. The third chapter, the title of which is Return on investment: The future of evidence based research on ICT-enhanced business English, written by Antonio J. jiménez-Muñoz, considers the need for sound evidence based research in the field of LSP. The author proposes that current multi-dimensional databases and online 
analytical processing permit management of complex data into $n$-dimensional hypercubes in order to accommodate diverse factors such as motivation, previous experience, ICT (information and communication technology) access, instructor ICT proficiency as well as the like as a particular added dimension significant to the context of education. Next, Chapter 4 entitled L2 English learning and performance through online activities: A case study, written by María Ángeles Escobar, offers a case study measuring outcomes of an online course task design, being adult learners the participants of the experiment in an English online course. The outcomes demonstrate not only that well-motivated students gain better results in foreign language testing but also that higher student motivation is due to "making the move" to the exemplified aspects of e-learning.

The four chapters in Part 2 are focused on languages and technology-enhanced assessment. This section offers 4 contributions which complement each other and, at the same time, the four chapters are based on the use of Computer-Assisted Language Testing (CALT) systems. In the first contribution, the title of which is Language testing in the digital era, written by Miguel Fernández Álvarez, analyzes various types of computerized assessment such as CBT (computer-based testing), CAT (computer adaptive testing) and WBT (web-based testing) which have given enormous benefits to assessment research by offering immediate feedback, ways to store test outcomes for deeper analysis, multimedia presentations, as well as examtaker's skill to pace on his/her own. The author proposes future directions in relation to the utilization of CALT in meeting the needs of latest generations of learners who are, indeed, rather familiar with portable electronic devices confirming the 100\% reliable exam taking. Vicente Beltrán-Palanques, in the second chapter, the title of which is Synchronous computer-mediated communication in ILP research: A study based on ESP context, offers a rather interesting research in the specialized language domain, English for Psychology. The author focuses on the outcomes whose origin lies in the retrospective verbal reports (RVRs) of the six participants who perform the speech act of advice, paying special attention to the aspects of language attended while planning and executing the speech act. The next contribution is the chapter written by Elena Domínguez Romero, Isabel de Armas Ranero and Ana FernándezPampillón Cesteros, which is called The COdA scoring rubric: An attempt to facilitate assessment in the field of digital educational materials in higher education. The authors try to re-examine and verify the need to produce quality specialized online academic materials in university settings through a scoring rubric in learning objects language tools which would improve the accuracy of the tool(s) while, at the same time, lowering the time span of the application. The updated model should function as the basis for a future standard that is, nowadays, being developed by the Spanish Association for Standardization and Certification (in Spanish, Asociación Española de 
Normalización y Certificación, AENOR) and which was to be completed by the end of the year 2016. The final chapter, entitled Enabling automatic, technology-enhanced assessment in language e-learning: Using ontologies and linguistic annotation merge to improve accuracy by Antonio Pareja Lora, demonstrates how to utilize ontologies and linguistic annotations with the purpose of interlinking various POS (parts of speech) taggers together into a prototype for producing combined POS. The author states that this leads to the creation of a set of linguistic tools which, on the one hand, should lower the POS tagging error rate while, on the other hand, the inaccuracy of the devised tools.

The third part of this volume encompasses three further chapters. This part is related to mobile-assisted language learning and its use in learning Languages for Specific Purposes. Joshua Underwood, in the first contribution, entitled Challenges and opportunities in enacting MALL designs for $L S P$, analyses designs for MALL, appropriate for learning LSPs and deals with the challenges in providing these MALL designs. In the second chapter, whose title is Designer learning: The teacher as designer of mobile-based classroom learning experiences, Nicky Hockly analyses a small-scale classroom based research which takes place at two different levels with students who are learning English as a Foreign Language in the UK at a two-week course. In the last contribution of this thematic section, Elena Bárcena, Timothy Read and Agnes Kukulska-Hulme, in Mobile and massive language learning, explore and enhance Language Massive Online Open Courses (LMOOCS) which give the students the chance to overcome the constraints of face-to-face in-class contact and to obtain benefits from the use of special devices such as portable course clients, mobilesensor-enabled devices and handheld computers.

The fourth part is composed of three chapters dealing with language massive open online courses (LMOOCS). Maggie Sokolik, in her chapter entitled Academic writing in MOOC environments: Challenges and rewards, analyses the challenges to academic writing in a MOOC environment. The author suggests that both teachers and students take great benefits from this approach offering opportunities to build communities of discourse, having access to courses and colleagues who are available further in distance, taking place in a relatively low-stress environment. Additionally, researchers might obtain access to large groups of learners as well as large databases of writing samples which might be utilized in order to develop improved models to develop written competence. The second contribution to this thematic section, Language MOOCs: Better by design written by Fernando Rubio, Carolin Fuchs and Edward Dixon, enhances the idea that designers of LMOOCs can further hold their learners accountable for their out of course engagements through various questionnaires, allowing thus students interact with each other in their in 
their personal learning environments. Moreover, the authors propose some advantages of utilizing questionnaires in order to gather data with the aim of improving teaching instructions. These surveys can be utilized, regardless of the format, to obtain data about: 1) the students' personal learning experiences in the MOOC; 2) their own considerations on how and what they learned, and 3) where they generally experience difficulties. With this information, both course designers and instructors have the chance to decide where modifications are necessary for improving content, creating more effective instruction and helping learners maximize their language learning process. The third and last chapter, the title of which is Enhancing specialized vocabulary through social learning in language MOOCs, written by Elena Martín-Monje and Patricia Ventura, try to shed light on the acquisition of specialized vocabulary (SV) in MOOCs focusing on how social learning can be promoted when connecting a social network such as Facebook to online language courses.

Part 5 deals with corpus-based approaches for specialized linguistic domains in three different chapters. In the first chapter of this thematic section, the title of which is Corpus-based teaching in LSP, Tony Berber Sardinha offers an overview on how corpus linguistics can be explored in the LSP classroom through analyses of two different corpora, being commercial aviation maintenance manuals and relevant research papers. Mario Carranza Díez, in the second contribution whose title is Transcription and annotation of non-native spoken corpora, describes the process of transcribing and annotating spontaneous non-native spoken corpora not only for the empirical research on second language pronunciation acquisition but also to develop a computer-assisted pronunciation training application. The final contributors of this section, María del Mar Sánchez Ramos and Francisco J. Vigier Moreno, in the chapter Using monolingual virtual corpora in public service legal translator training, present the utilization of monolingual virtual corpora in public service legal translator training, suggesting that corpus management instruments can be successfully used for helping the learners acquire high level of expertise in this specific language field.

Finally and lastly, Part 6 covers three chapters which are related to the computerassisted translation tools for language learning. The first chapter, the title of which is Computer-assisted translation tools as tools for language learning by María Fernández Parra, analyzes Computer Assisted Translation (CAT) tools, along with their features and applications highlighting, at least, two relevant benefits of such tools for language learning. These significant advantages are portability and shareability. These benefits are promoted by their ability of reuse, being opposed to other kinds of computerized tools. The second contribution to this thematic section is the 
chapter entitled Applying corpora-based translation studies to the classroom: Languages for specific purposes acquisition by Montserrat Bermúdez Bausela. The author focuses on the utilization of corpora not only in translation studies but also in the application of "ad hoc corpora" in LSP. The last chapter of this thematic section, whose title is VISP: A MALL-based app using audio description techniques to improve B1 EFL students' oral competence, and written by Ana Ibáñez Moreno and Anna Vermeulen, offers empirical data on how a MALL-based app including audio description techniques can improve the learners' oral competence. The findings shown demonstrate the positive and promising potential of using audio description (AD) to a mobile app which is aimed at enhancing oral skills in the foreign language learning experience.

The six thematic sections of this book follow a similar pattern: The first contribution, in each part, explains the topic from a theoretical perspective while the other chapters provide empirical evidence of the progress made in the field. Moreover, they are closely interconnected, revealing all those subjacent topics generating the kaleidoscopic view which Jozef Colpaert refers to in his foreword (xvii-xix). Additionally, assessment proves to be relevant in language learning, being treated in every section. This book also shows how closely related some of the fields are. There is a natural connection between corpus-based approaches to specialized linguistic domains and computer-assisted translation tools for language learning.

To sum up, the book Technology-Enhanced Language Learning for Specialized Domains: Practical Applications and Mobility, edited by Elena Martín-Monje, Izaskun Elorza \& Blanca García Riaza, covers the main fields in which LSP and technological applications are progressing hand in hand, for the time being and most likely for the near future. This volume makes an in-depth analysis and enhances the idea that implying in technology-enhanced language learning (TELL) is constantly a challenge which requires time and commitment. In the recent established era in the area of language teaching, both teachers and learners in specialized domains, as the ones described in this book, utilize technology so that this educational technology is integrated as part of their everyday behaviour for experiencing the world and realities around them. The readers of this book can perfectly understand, from the different contributions in the current volume, that not only research but also practice in the field of TELL propose that if and when adequately implemented, TELL can offer a significant contribution to these aspects: experiential learning, motivation, enhanced student achievement, authentic study materials, greater interaction, individualization, independence from a single source of information, and other related advantages. 
This volume, Technology-Enhanced Language Learning for Specialized Domains, is suggested to both teachers and learners in the area of processing Language for Specific Purposes (LSP) since it is truly valuable as a resource to the numerous possibilities in order to explore the latest trends of TELL for specialized domains in the future, with the aim of exploiting fully the consequently arising perks and privileges in the field.

\section{References}

Arnó Maciá, E., A. Soler Cervera and C. Rueda Ramos (eds) (2006). Information Technology in Languages forSpecific Purposes. Issues and Prospects. New York: Springer. 